\title{
Coxiella Detection in Ticks from Wildlife and Livestock in Malaysia
}

\author{
Jing-Jing Khoo, Fang-Shiang Lim,,2 Fezshin Chen,,2 Wai-Hong Phoon,,2 Chee-Sieng Khor, \\ Brian L. Pike, Li-Yen Chang, ${ }^{1,2}$ and Sazaly AbuBakar ${ }^{1,2}$
}

\begin{abstract}
Recent studies have shown that ticks harbor Coxiella-like bacteria, which are potentially tick-specific endosymbionts. We recently described the detection of Coxiella-like bacteria and possibly Coxiella burnetii in ticks found from rural areas in Malaysia. In the present study, we collected ticks, including Haemaphysalis bispinosa, Haemaphysalis hystricis, Dermacentor compactus, Dermacentor steini, and Amblyomma sp. from wildlife and domesticated goats from four different locations in Malaysia. Coxiella 16s rRNA genomic sequences were detected by PCR in $89 \%$ of ticks tested. Similarity analysis and phylogenetic analyses of the $16 s$ rRNA and rpoB partial sequences were performed for 10 representative samples selected based on the tick species, sex, and location. The findings here suggested the presence of $C$. burnetii in two samples, each from D. steini and $H$. hystricis. The sequences of both samples clustered with published $C$. burnetii sequences. The remaining eight tick samples were shown to harbor 16s rRNA sequences of Coxiella-like bacteria, which clustered phylogenetically according to the respective tick host species. The findings presented here added to the growing evidence of the association between Coxiella-like bacteria and ticks across species and geographical boundaries. The importance of $C$. burnetii found in ticks in Malaysia warrants further investigation.
\end{abstract}

Keywords: Coxiella, domestic animals, ticks, zoonotic

\section{Introduction}

T ICKS ARE EFFECTIVE VECTORS for a range of infectious diseases. A number of hard and soft tick species, including Amblyomma, Dermacentor, Haemaphysalis, Hyalomma, Rhipicephalus, and Ornithodoros, have been documented to harbor the Coxiella burnetii (Špitalská and Kocianová 2003, Mediannikov et al. 2010, Cooper et al. 2012). C. burnetii is the causative agent for $\mathrm{Q}$ fever, a worldwide zoonotic disease commonly affecting livestock animals such as cattle, goats, and sheep. Human infections are most likely due to contact with excreta from these animals or via the inhalation of contaminated aerosols (Tissot-Dupont and Raoult 2008). The role of ticks in transmitting Q fever to humans and animals has not been established, even though ticks are observed to be competent vectors for the transmission of $C$. burnetii to mammalian hosts in the laboratory setting (Duron et al. 2015). However, a high prevalence of $C$. burnetii in ticks in some endemic regions, such as
West Africa, may indicate a role for ticks in the epidemiology of Q fever (Mediannikov et al. 2010).

A number of studies have identified a family of tick endosymbionts that are closely related to $C$. burnetii. These endosymbionts have been found in a variety of ticks species, including Haemaphysalis (Lee et al. 2004, Ahantarig et al. 2011, Arthan et al. 2015), Amblyomma (Klyachko et al. 2007, Machado-Ferreira et al. 2011), Rhipicephalus (Bernasconi et al. 2002), Ixodes (Kurtti et al. 2002), Ornithodoros (Almeida et al. 2012, Duron et al. 2014), and Argas (Reeves 2008). The most recent study based on multilocus sequence analysis of a few housekeeping genes demonstrated that all Coxiella strains cluster into four highly divergent clades, suggesting that $C$. burnetii evolved from a tick endosymbiont (Duron et al. 2015). Studies have indicated that these Coxiella endosymbionts may be important in providing for the vitamin and cofactor biosynthesis pathways and in determining the reproductive fitness of the tick hosts (Jasinskas

\footnotetext{
${ }^{1}$ Tropical Infectious Diseases Research and Education Centre (TIDREC), University of Malaya, Kuala Lumpur, Malaysia.

${ }^{2}$ Department of Medical Microbiology, Faculty of Medicine, University of Malaya, Kuala Lumpur, Malaysia.

${ }^{3}$ Naval Medical Research Center-Asia (NMRC-A), Singapore, Singapore.
}

(C) Jing-Jing Khoo, et al., 2016; Published by Mary Ann Liebert, Inc. This Open Access article is distributed under the terms of the Creative Commons Attribution Noncommercial License (http://creativecommons.org/licenses/by-nc/4.0/) which permits any noncommercial use, distribution, and reproduction in any medium, provided the original author(s) and the source are credited. 
et al. 2007, Zhong et al. 2007). Therefore, understanding the role of Coxiella endosymbionts in maintaining tick growth and survival may yield novel approaches in the control and management of tick populations.

In Malaysia, human populations living in the forested or rural regions, including farmers and the indigenous people of Malaysia, the Orang Asli, are at risk of infection from tickborne pathogens (Audy et al. 1960, Paramasvaran et al. 2009). Unpublished seroprevalence data from our laboratory were indicative of past infections with $C$. burnetii among the Orang Asli populations. However, the prevalence of C. burnetii and Coxiella-like bacteria associated with ticks from the forests or rural areas in Malaysia has never been fully investigated. In this study, we performed molecular detection of Coxiella bacteria from ticks collected from wildlife and farm areas from selected sites in Malaysia.

\section{Materials and Methods}

\section{Tick samples}

Ticks were collected with the assistance from the Orang Asli from the carcasses of wild animals (wild boars and a single porcupine) obtained from routine hunting trips in the state of Selangor $\left(3.0738^{\circ} \mathrm{N}, 101.5183^{\circ} \mathrm{E}\right)$. All site visits to the Orang Asli villages were performed with the approval from the Department of Orang Asli Development, Malaysia (JAKOA). Ticks were also collected from goats found in privately owned farms located in two locations in the state of Perak $\left(4.5921^{\circ} \mathrm{N}, 101.0901^{\circ} \mathrm{E}\right)$, and one location in the state of Negeri Sembilan $\left(2.7258^{\circ} \mathrm{N}, 101.9424^{\circ} \mathrm{E}\right)$, with the permission from the respective farm owners. All goat farms were managed by semi-intensive grazing system, in which the grazing area includes palm oil and rubber plantations as well as secondary forests. Once removed from the hosts, the ticks were kept alive in ziplock bags for transportation to the laboratory within $24 \mathrm{~h}$ and stored in $-80^{\circ} \mathrm{C}$ until further processing. All sampling was performed during the time from July 2014 to July 2015. The collected ticks were microscopically identified and classified by life stage using published taxonomic keys (Wassef and Hoogstraal 1983, 1986, Tanskul and Inlao 1989). For DNA extraction, frozen tick samples were thawed and washed rigorously three times in $70 \%$ ethanol followed by sterile deionized water to remove possible environmental contaminants.

\section{DNA extraction from tick samples}

Mortars and pestles were soaked in $10 \%$ bleach for $1 \mathrm{~h}$, thoroughly rinsed with sterile deionized water, and sterilized at $160^{\circ} \mathrm{C}$ overnight to ensure the absence of contaminating materials. Swabs were taken and amplification of bacterial $16 \mathrm{~s} r R N A$ sequences was performed to ensure no residual genomic material remained (Khoo et al. 2016). Tick samples were ground in liquid nitrogen using chilled mortar and pestle in a biosafety cabinet, using separate sets of mortar and pestle for each sample. The resulting finely ground sample was resuspended in $500 \mu \mathrm{L}$ of sterile phosphate-buffered saline (PBS). DNA was extracted from a $200 \mu \mathrm{L}$ aliquot of the suspension using the QIAamp DNA Mini Kit (Qiagen, Hilden, Germany) according to the manufacturer's protocol. Mock extractions on PBS alone were performed in parallel and the resulting DNA was subjected to further screening as below.
Polymerase chain reaction for ticks and Coxiella gene detection

PCR amplification of partial mitochondrial 16s rRNA sequence of selected ticks was performed using primers and protocols previously described (Black and Piesman 1994) for Haemaphysalis identification. Coxiella sp. was detected by a nested-PCR protocol to amplify the $16 \mathrm{~s} r R N A$ partial gene sequence using primers previously described (Duron et al. 2014) together with a separate pair of nested primers (forward: 5'-ATTTCCGGTGTAGCGGTG-3' and reverse: 5'ACGACAGCCATGCAGCAC-3'). Samples exhibiting the amplification of all fragments during nested PCR were considered as positive for the presence of the Coxiella DNA. Samples displaying no amplification of the Coxiella-specific 16s rRNA fragment were subjected to additional PCR amplification of nonspecific bacterial $16 \mathrm{~s}$ rRNA gene as described in an earlier study (Khoo et al. 2016). The partial sequence of the $r p o B$ gene from selected samples was amplified using previously described primers and protocols (Duron et al. 2015). Amplicons from representative positive samples were selected, gel purified, and sequenced based on the criteria of species, sex, and location. Mock extractions were also tested in the nested-PCR procedures, which did not exhibit any amplification of the targeted genes.

\section{Sequence analysis}

The sequences obtained were compared to the available sequences in the NCBI GenBank database (www.ncbi.nml. nih.gov/BLAST/). Primer-trimmed sequences of Coxiella sp. 16s $r R N A$ and $r p o B$ genes were aligned using CLUSTALW, as implemented in MEGA6 (Tamura et al. 2013). Poorly aligned positions were removed using GBLOCKS (Talavera and Castresana 2007). Genetic variation between each sample was determined by computing pairwise nucleotide differences in MEGA6. All sequences obtained were deposited in the European Nucleotide Archive (ENA) (Accession Numbers: LT593104-LT593134 for tick mitochondrial $16 \mathrm{~s}$ rRNAs, LT009428-LT009437 for Coxiella 16s rRNAs, and LT174608-LT174617 for Coxiella rpoB).

\section{Phylogenetic analysis}

Phylogenies were inferred using the Bayesian Markov chain Monte Carlo method implemented in BEAST version 1.8.2 (Drummond and Rambaut 2007). The best-fit model of nucleotide substitution was selected by Akaike Information Criterion as implemented in jModelTest 2.1.7 (Posada 2008). $\mathrm{GTR}+\mathrm{I}+\mathrm{G}$ and $\mathrm{GTR}+\mathrm{G}$ were selected, respectively, for $16 \mathrm{~s}$ $r R N A$ and $r p o B$ phylogenies.

\section{Results}

\section{Tick samples}

In this study, 55 ticks were collected from four separate locations in Malaysia. The number of ticks and collected data are summarized in Table 1. From the Orang Asli village in Selangor, 34 adult ticks were collected. These ticks were identified as Haemaphysalis hystricis (13 males, 6 females), Dermacentor steini (6 males, 1 female), Dermacentor compactus (2 males), and Amblyomma sp. (6 females). All H. hystricis and Dermacentor ticks were collected from the 
Table 1. Coxiella Detection in Ticks Collected from Various Locations in Malaysia USING $16 s$ RRNA GENE AMPLIFICATION

\begin{tabular}{|c|c|c|c|c|c|c|c|}
\hline Location & Host & Species & Stage & $\operatorname{Sex}$ & Engorged & $\begin{array}{c}\text { Total } \\
\text { number }\end{array}$ & $\begin{array}{l}\text { PCR positive for Coxiella } \\
\text { partial 16s rRNA }(n=x)\end{array}$ \\
\hline \multirow[t]{7}{*}{ Selangor } & \multirow[t]{2}{*}{ Wild boar } & \multirow[t]{2}{*}{ Haemaphysalis hystricis } & \multirow{2}{*}{ Adult } & $\mathrm{F}$ & No & 6 & 6 \\
\hline & & & & M & No & 13 & 13 \\
\hline & \multirow{2}{*}{ Wild boar } & \multirow{2}{*}{ Dermacentor steini } & \multirow{2}{*}{ Adult } & $\mathrm{F}$ & No & 1 & 0 \\
\hline & & & & M & No & 6 & 2 \\
\hline & Wild boar & Dermacentor compactus & Adult & M & No & 2 & 1 \\
\hline & \multirow[t]{2}{*}{ Porcupine } & \multirow[t]{2}{*}{ Amblyomma sp. } & \multirow[t]{2}{*}{ Adult } & \multirow[t]{2}{*}{$\mathrm{F}$} & Yes & 5 & 5 \\
\hline & & & & & No & 1 & 1 \\
\hline \multirow[t]{2}{*}{ Perak—Farm B } & \multirow[t]{2}{*}{ Goats } & \multirow[t]{2}{*}{ Haemaphysalis bispinosa } & \multirow[t]{2}{*}{ Adult } & \multirow[t]{2}{*}{$\mathrm{F}$} & Yes & 2 & 2 \\
\hline & & & & & No & 7 & 7 \\
\hline \multirow[t]{3}{*}{ Perak-Farm T } & \multirow[t]{3}{*}{ Goats } & \multirow[t]{3}{*}{ H. bispinosa } & \multirow{3}{*}{ Adult } & $\mathrm{F}$ & Yes & 3 & 3 \\
\hline & & & & & No & 2 & 2 \\
\hline & & & & M & No & 2 & 2 \\
\hline \multirow[t]{3}{*}{ Negeri Sembilan } & \multirow[t]{3}{*}{ Goats } & \multirow[t]{2}{*}{ H. bispinosa } & Adult & $\mathrm{F}$ & No & 4 & 4 \\
\hline & & & Nymph & & No & 1 & 1 \\
\hline & & Total & & & & 55 & 49 \\
\hline
\end{tabular}

carcasses of two wild boars. All Amblyomma ticks were collected from a single porcupine. Ticks ( 2 males, 18 females) collected from the goat farms in Perak and Negeri Sembilan were all identified as adult Haemaphysalis bispinosa. A single nymph was also identified as $H$. bispinosa. A number of female ticks were observed to be engorged: $A m$ blyomma (5/6), H. bispinosa from Farm B (2/9) and Farm T (3/5) in Perak. The partial mitochondrial 16s $r R N A$ sequences amplified from Haemaphysalis ticks in Selangor and Perak were 99\%-100\% identical to H. hystricis (NCBI Accession No.: AB819197.1) and H. bispinosa (NCBI Accession No.: KC853419.1), respectively (Supplementary Table S1; Supplementary Data are available online at www.liebertpub .com/vbz), which is consistent with the morphological identifications.

\section{Detection of Coxiella in tick samples}

Overall, $89 \%(49 / 55)$ of the ticks in this study were positive for Coxiella 16s rRNA genomic sequences (Table 1). All Haemaphysalis ticks tested, regardless of species, sex, location, or engorgement for females, exhibited positive Coxiella genomic sequences by PCR (40/40). Coxiella was also detected in all Amblyomma ticks tested (6/6), 2/6 of male D. steini, but not in the female tested $(0 / 1)$. One out of the two D. compactus in the study was PCR positive for Coxiella. Nonspecific bacterial 16s rRNA genes were amplified from all samples that tested negative for Coxiella 16s rRNA PCR (data not shown), indicating that the absence of the amplification of Coxiella 16s rRNA was not due to compromised sample DNA quality.

\section{Analyses of tick-derived Coxiella 16s rRNA partial sequences}

Ten representative samples, selected from nonengorged samples, were selected based on species, location of sampling, and sex (summarized in Table 2) to be sequenced for the Coxiella partial 16s rRNA sequences. The similarity matrix resulting from a pairwise nucleotide comparison between all samples in this study, together with two $C$. burnetii reference strains, is presented in Table 3. All samples in this study, except for S009, S012, and S014, displayed $96.2 \%$ to $96.7 \%$ similarity to the $C$. burnetii reference strains. Coxiella sequences from $H$. bispinosa (N002, B002, T007, and T008) were $98.2 \%$ to $99.8 \%$ similar to each other. S002 and S006, both from $H$. hystricis, shared $99.9 \%$ sequence similarity. S014 from $H$. hystricis and S009 from D. steini also shared $99.9 \%$ sequence similarity to each other, but displayed only $96.2 \%$ to $96.7 \%$ similarity to other samples in this study. S012, from D. compactus, displayed $94.2 \%$ to $94.7 \%$ similarity to all other samples. S027, from Amblyomma, displayed only $96.6 \%$ to $96.9 \%$ sequence similarity to the reference strains and the other samples here.

\section{Phylogeny of tick-derived Coxiella partial 16s rRNA and rpoB genes}

A Bayesian-inferred phylogenetic tree was constructed based on the partial 16s rRNA sequences from this study and Coxiella sequences derived from the NCBI GenBank (Fig. 1, NCBI accession numbers tabulated in Supplementary Table S2). The published sequences were selected to reflect the previously reported clustering of Coxiella strains into four highly divergent clades according to the tick hosts (Duron et al. 2015). Clade A, comprising various $C$. burnetii isolates and Coxiella of the

Table 2. Representative Coxiella Sequences Used in Phylogenetic Analysis

\begin{tabular}{llll}
\hline $\begin{array}{l}\text { Sample } \\
\text { number }\end{array}$ & \multicolumn{1}{c}{ Tick species } & Sex & \multicolumn{1}{c}{ Location } \\
\hline N002 & H. bispinosa & $\mathrm{F}$ & Negeri Sembilan \\
B002 & H. bispinosa & $\mathrm{F}$ & Perak-Farm B \\
T007 & H. bispinosa & $\mathrm{F}$ & Perak-Farm T \\
T008 & H. bispinosa & $\mathrm{M}$ & Perak-Farm T \\
S002 & H. hystricis & $\mathrm{F}$ & Selangor \\
S006 & H. hystricis & $\mathrm{M}$ & Selangor \\
S014 & H. hystricis & $\mathrm{F}$ & Selangor \\
S009 & D. steini & $\mathrm{M}$ & Selangor \\
S012 & D. compactus & $\mathrm{M}$ & Selangor \\
S027 & Amblyomma sp. & $\mathrm{F}$ & Selangor \\
\hline
\end{tabular}


Table 3. Similarity Matrix of Coxiella Partial 16s RRNA Sequences

\begin{tabular}{|c|c|c|c|c|c|c|c|c|c|c|c|c|}
\hline & 1 & 2 & 3 & 4 & 5 & 6 & 7 & 8 & 9 & 10 & 11 & 12 \\
\hline 1 Coxiella burnetii RSA 493 & 100.0 & 99.9 & 96.4 & 96.4 & 96.4 & 96.2 & 96.7 & 96.7 & 100.0 & 99.9 & 94.8 & 3 \\
\hline 2 C. burnetii CbuG Q212 & & 100.0 & 96.3 & 96.3 & 96.3 & 96.1 & 96.6 & 96.7 & 99.9 & 99.8 & 94.9 & \\
\hline 3 Coxiella in $H$. bispinosa $\mathrm{N} 002$ & & & 100.0 & 99.8 & 99.8 & 99.7 & 98.3 & 98.4 & 96.4 & 96.3 & 94.3 & \\
\hline 4 Coxiella in $H$. bispinosa $\mathrm{B} 002$ & & & & 100.0 & 99.8 & 99.8 & 98.3 & 98.4 & 96.4 & 96.3 & 94.3 & \\
\hline 5 Coxiella in H. bispinosa T007 & & & & & 100.0 & 99.7 & 98.3 & 98.4 & 96.4 & 96.3 & 94.3 & \\
\hline 6 Coxiella in H. bispinosa T008 & & & & & & 100.0 & 98.3 & 98.2 & 96.2 & 96.3 & 94.2 & \\
\hline 7 Coxiella in H. hystricis S002 & & & & & & & 100.0 & 99.9 & 96.7 & 96.7 & 94.4 & 96 \\
\hline 8 Coxiella in $H$. hystricis S006 & & & & & & & & 100.0 & 96.7 & 96.7 & 94.5 & \\
\hline 9 Coxiella in H. hystricis S014 & & & & & & & & & 100.0 & 99.9 & 94.8 & 96 \\
\hline 10 Coxiella in D. steini S009 & & & & & & & & & & 100.0 & 94.7 & \\
\hline 11 Coxiella in D. compactus S012 & & & & & & & & & & & 100.0 & 94 \\
\hline 12 Coxiella in Amblyomma sp. S027 & & & & & & & & & & & & 100.0 \\
\hline
\end{tabular}

The similarity of the $16 \mathrm{~s}$ rRNA sequence based on the comparison of 1165 nucleotide positions from the Coxiella reference and sample strains.

Ornithodoros soft ticks, as well as Clade B, consisting of Coxiella from Amblyomma variegatum and Ixodes sp., was clearly seen in our tree. Clade C comprising Coxiella from Rhipicephalus and Clade D comprising Coxiella Haemaphysalis and Dermacentor were also seen. However, Coxiella from Amblyomma americanum and Amblyomma cajennense, which hosted Clade D in the previous study, were separated from the other Coxiella strains (Duron et al. 2015). It is important to note that the separation between the members of Clades $C$ and $D$ was only partially supported (posterior probability of 0.67 ). Hence, the phylogenetic tree based on 16s rRNA partial sequence alone may not be effective in resolving Clades $\mathrm{C}$ and $\mathrm{D}$.

Coxiella from D. steini (S009) and H. hystricis (S014) were clustered with various $C$. burnetii isolates in Clade A. Coxiella from H. hystricis (S002 and S006) were clustered with the Coxiella-like bacteria from Haemaphysalis longicornis. Coxiella from H. bispinosa (N002, B002, T007, and T008) clustered with the Coxiella from Haemaphysalis shimoga. S027 from Amblyomma sp. clustered with Coxiella from Ixodes uriae and A. variegatum. The Coxiella endosymbionts from Haemaphysalis, I. Uriae, and A. variegatum appeared to form Clade B together. S012 from D. compactus appeared to be distinct from other Coxiella strains tested here.

As a complementary analysis, a separate phylogenetic tree was constructed based on the partial sequence of the rроB gene (Fig. 2). The rpoB phylogeny showed S009 and S014 clustered with the other $C$. burnetii reference strains, which formed Clade A with the Ornithodoros rostratus endosymbionts. This finding was similar to the 16s rRNA phylogenetic tree. Coxiella from the Haemaphysalis ticks (N002, B002, T007, T008, S002, and S006) and Amblyomma sp. (S027) clustered into Clade B along with the endosymbionts from I. uriae and A. variegatum, also consistent with the $16 \mathrm{~s} r R N A$ phylogenetic tree. However, Coxiella from D. compactus (S012) clustered with the Coxiella from H. punctata based on the rpoB phylogeny into a collapsed $\mathrm{C}$ and $\mathrm{D}$ Clade.

\section{Discussion and Conclusion}

In the present study, we detected the presence of Coxiella genomic sequence in a number of tick species, including H. bispinosa, H. hystricis, D. compactus, D. steini, and Amblyomma sp., collected from wildlife and goats in Malaysia.
The results are consistent with reports identifying Coxiellalike bacteria or endosymbionts in a number of Haemaphysalis, Dermacentor, and Amblyomma tick species (Lee et al. 2004, Jasinskas et al. 2007, Klyachko et al. 2007, Liu et al. 2013, Arthan et al. 2015, Duron et al. 2015). Several female ticks of Haemaphysalis and Amblyomma sampled here were fully engorged, hence it is possible that any Coxiella DNA observed may have originated from host blood. However, the detection of Coxiella sequences in most of the nonengorged ticks suggests the association of Coxiella linked to the ticks themselves and not the animal host. Thus, only nonengorged tick samples were chosen as representative samples for sequencing and further analyses to avoid potential confounding effects from host blood.

In pairwise nucleotide comparisons of the eight Coxiellalike bacteria sequenced here, Coxiella from the same genus of ticks, such as Haemaphysalis, exhibited greater similarity to each other, suggesting that genetic variation is host dependent. Although Duron et al. (2015) have reported that Coxiella endosymbionts from Haemaphysalis belong to Clade D, our findings suggested that Coxiella-like bacteria from $H$. hystricis, $H$. bispinosa, $H$. shimoga, and $H$. longicornis are clustered together with Coxiella endosymbionts from A. variegatum, I. uriae, and the Amblyomma sp. (S027) in Clade B. It is important to note, however, that the Haemaphysalis hosts for the Coxiella strains clustered in Clade B in this study are primarily found in the Asian or Southeast Asian regions, which were not represented in the study by Duron et al. (2015). H. punctata, the only Haemaphysalis representative in Duron's study, commonly occupies the European continent. Since Coxiella-like bacteria are likely to have coevolved with the tick hosts, the geographical separation and the subsequent evolutionary history of ticks could have resulted in the genetic differences as shown in the phylogenies. This observation is also mirrored in the separation of the Coxiella strains from Amblyomma ticks into different clades (Duron et al. 2015). The Coxiella from A. americanum and A. cajennense, both dominantly found in the Americas, are clustered in Clade D. A. variegatum, which is prevalent in Africa, belongs to Clade B.

In this study, the phylogenies based on the 16s rRNA or $r p o B$ sequences individually were insufficient to separate the members of Clades $\mathrm{C}$ and $\mathrm{D}$. These discrepancies may have 


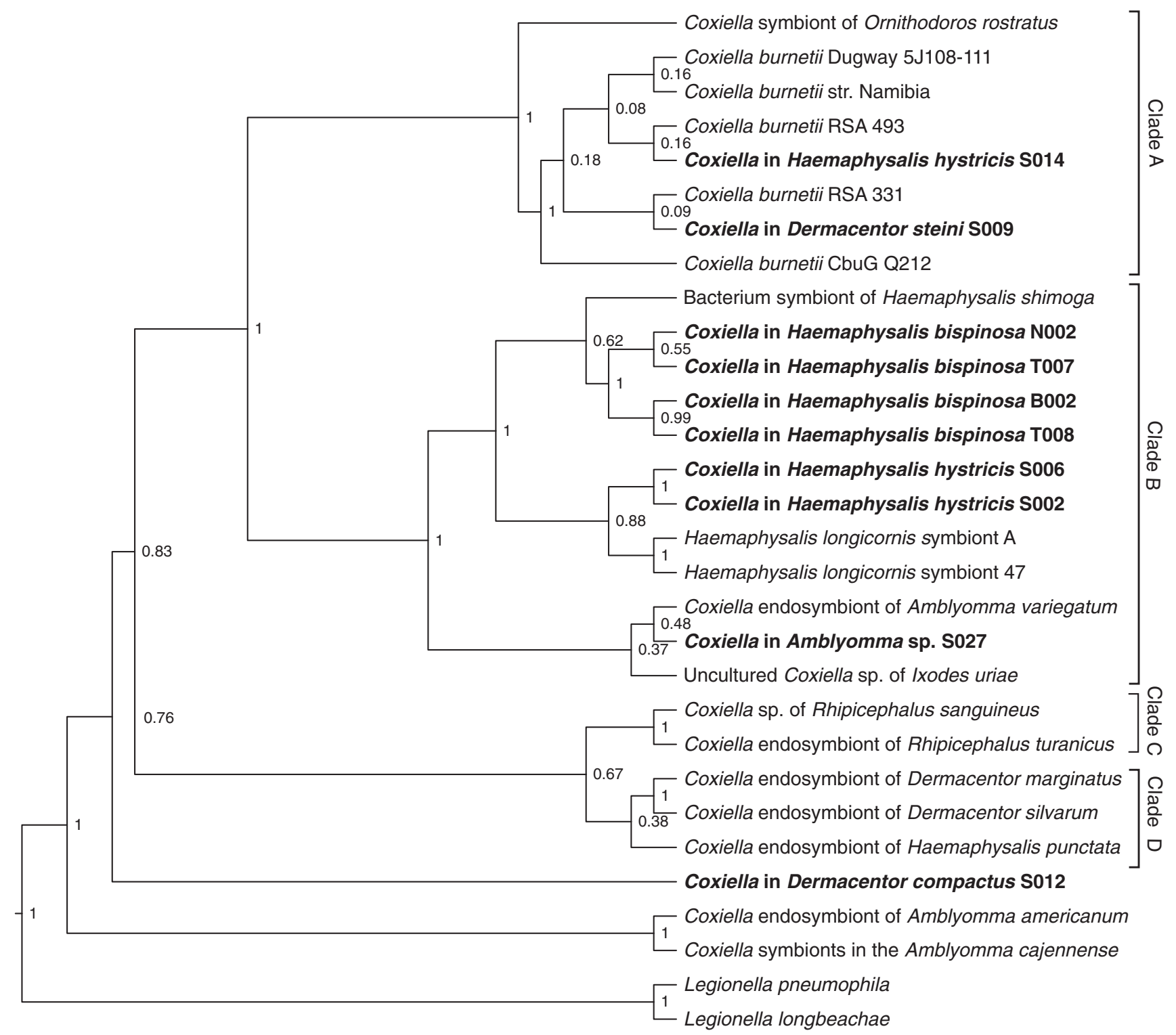

FIG. 1. Phylogenetic tree of Coxiella sp. based on partial $16 s \mathrm{~s} R A$ sequences. Bayesian-inferred phylogenetic tree of Coxiella sp. based on 1165 aligned nucleotides of the 16s rRNA sequence. The posterior probabilities are displayed adjacent to each node. Samples from this study are highlighted in bold. Clades labeled are indicative of the clustering of various Coxiella strains previously described by Duron et al. (2015). Sequences from Legionella pneumophila and Legionella longbeachae were included as the outgroup.

arisen due to the use of individual genes in the phylogenetic analyses here as opposed to the multilocus approach of the previous study, which used five concatenated gene sequences in the phylogenetic analyses (Duron et al. 2015). The singlegene analyses used here may also explain the inconsistencies observed for the Coxiella from D. compactus (S012) in the $16 s$ rRNA and $r p o B$ phylogenies. The genetic relationship of this strain will need to be confirmed with additional analyses, including the use of the multilocus sequencing approach.

The Coxiella sequences obtained in this study were directly sequenced from PCR amplicons, which excluded the possibility for investigating coinfections of multiple species of Coxiella, including coinfections of C. burnetii and Coxiellalike bacteria. Recent studies have shown that coinfections of pathogens and endosymbionts in individual ticks were com- mon especially in Ixodes ricinus ticks (Moutailler et al. 2016). At the time of writing, the authors were unaware of studies investigating the coinfections of Coxiella species in individual ticks, which merits further investigation as it will be important to study the functional interactions between potentially symbiotic Coxiella strains and the pathogenic C. burnetii.

Several studies have investigated the presence of Coxiellalike bacteria or endosymbionts in different tick species based on the detection of the $16 \mathrm{~s}$ rRNA gene. A. americanum, A. cajennense, O. rostratus, and Ornithodoros capensis appeared to display high frequency for the presence of Coxiella bacteria (Clay et al. 2008, Machado-Ferreira et al. 2011, Almeida et al. 2012, Duron et al. 2014). On the contrary, other tick species, such as a number of Haemaphysalis and Rhipicephalus species, showed variable or low prevalence of 


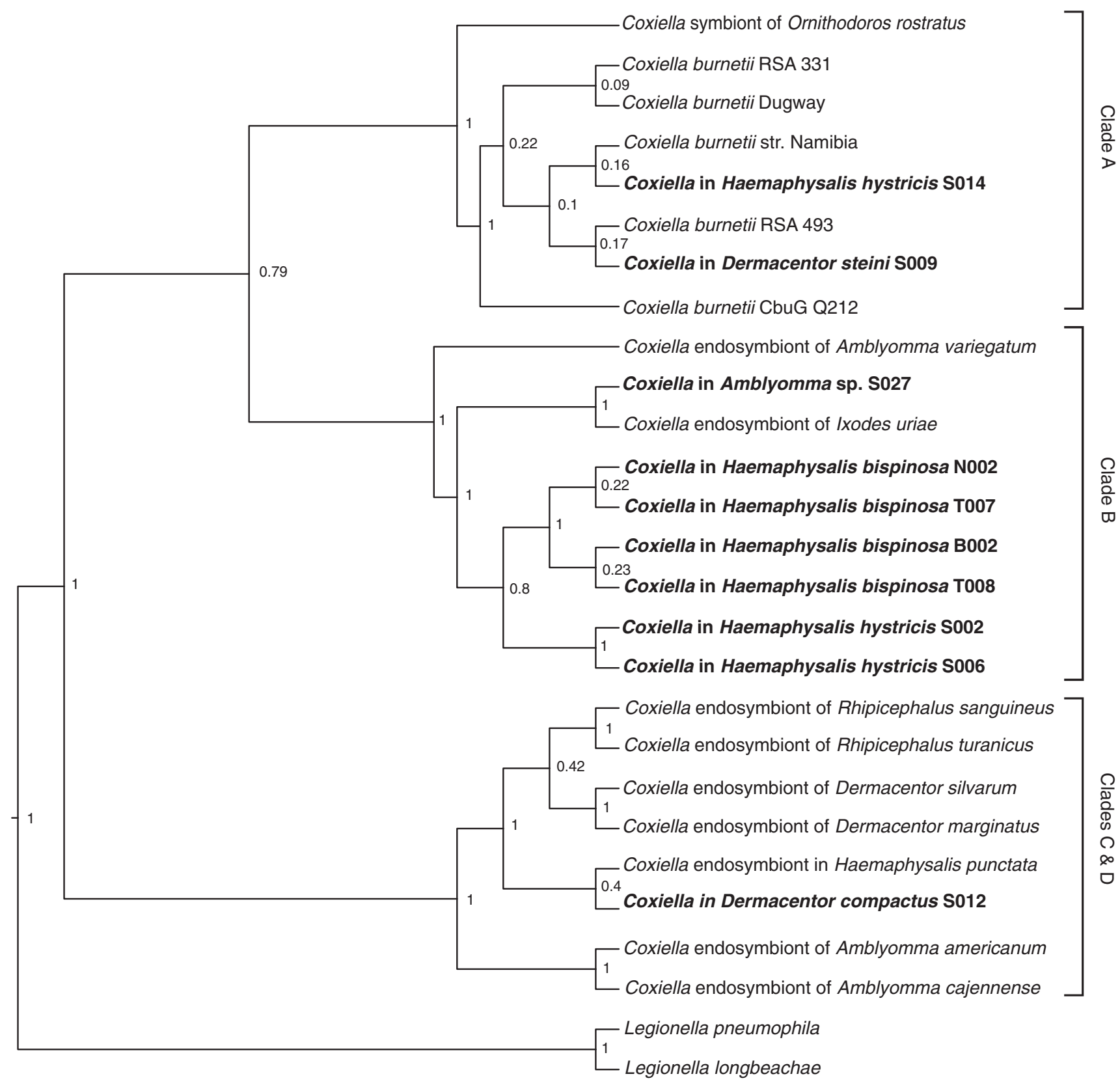

FIG. 2. Phylogenetic tree of Coxiella sp. based on partial rpoB sequences. Bayesian-inferred phylogenetic tree of Coxiella sp. based on 485 aligned nucleotides of the $r p o B$ sequence. The posterior probabilities are displayed adjacent to each node. Samples from this study are highlighted in bold. Clades labeled are indicative of the clustering of various Coxiella strains previously described by Duron et al. (2015). Sequences from L. pneumophila and L. longbeachae were included as the outgroup.

Coxiella-like bacteria (Bernasconi et al. 2002, Arthan et al. 2015). Our findings showed that all $H$. hystricis examined harbored Coxiella 16s rRNA sequence, whereas the prevalence was only $17 \%$ in $H$. hystricis ticks in previous reports (Arthan et al. 2015). The reason for this discrepancy is unclear. It is, however, important to note that Coxiella-like bacteria were not detected in all Haemaphysalis tick species examined in the prior study (Arthan et al. 2015). Similar observations were made in our previous attempt to study the bacterial communities in Haemaphysalis ticks in Malaysia (Khoo et al. 2016). We observed nonuniform relative abundance of Coxiella in all of the Haemaphysalis ticks tested. These findings suggest that the role of the Coxiella-like bacteria or endosymbiont may not be equally important across all tick species. Since the bulk of current studies into the physiological role of Coxiella endosymbiosis in ticks are investigated in A. americanum, extra precautions must be exercised when extrapolating the findings to other tick species (Klyachko et al. 2007, Zhong et al. 2007, Smith et al. 2015).

Overall, the findings here are consistent with the current hypothesis in which the association between Coxiella and ticks is universal across species and geographical boundaries (Duron et al. 2015). There are yet to be any reports of human or animal infections with Coxiella-like bacteria from ticks. 
However, avian infections of Coxiella-like bacteria have been reported even though there is no established link to ticks (Shivaprasad et al. 2008, Vapniarsky et al. 2012). Therefore, the potential of these Coxiella-like bacteria causing zoonotic infections merits further investigations.

Our findings suggest the possibility of $C$. burnetii infections in $H$. hystricis and D. steini ticks found in the forests of Malaysia, based on the clustering of the derived Coxiella sequences with other $C$. burnetii isolates in Clade A. To the best of the authors' knowledge, there is yet to be any report on the presence of $C$. burnetii infections in $H$. hystricis and D. steini ticks. Previous studies have identified the presence of $C$. burnetii in $R$. sanguineus ticks collected from dogs visiting a veterinary hospital in Malaysia (Watanabe et al. 2015). Wildlife animals such as wild boars and rodents have been previously implicated as reservoirs to infectious agents of livestock animals, including Brucella suis, C. burnetii, Anaplasma phagocytophilum, and Leptospira interrogans (Meng et al. 2009, Meerburg and Reusken 2011, Silaghi et al. 2014). The role of ticks in transmitting infection between multiple host animals and maintaining the reservoir of these pathogens merits investigation. The findings here also underscore the need for extensive prevalence studies of $C$. burnetii in wildlife within this region. Q fever outbreaks in livestock have been reported in Malaysia as recently as 2009 (Bina Rai et al. 2011, Norina et al. 2011). In humans, Q fever and the seroprevalence of $C$. burnetii have been reported in rural areas in Malaysia and Thailand (Tay et al. 1998, Suputtamongkol et al. 2003, Bina Rai et al. 2011, Blacksell et al. 2015). Unpublished serological studies performed on the Orang Asli in our laboratory have also indicated past C. burnetii exposure in this population. Hence, the presence of $C$. burnetii in wildlife animals and ticks is a potential health threat to both humans and livestock in this region.

In summary, the present study shows the presence of Coxiella-like bacteria, and possibly C. burnetii, in a number of tick species found on wildlife and domesticated goats from the rural areas in Malaysia. Our findings add to the growing evidence of the universality of Coxiella as tick-associated bacteria across various tick species and geographical locations. The possible presence of $C$. burnetii also suggests that ticks and the wildlife in Malaysia are potential reservoirs for the pathogen, signifying the need for improved surveillance activity to safeguard humans and livestock from the health risks of $\mathrm{Q}$ fever.

\section{Acknowledgments}

This study was supported, in part, by the research grants from the Ministry of Higher Education (MOHE) under the Fundamental Research Grant Scheme (FRGS, FP033-2014A), the High Impact Research (HIR)-MOHE Grant (E000013-20001), University of Malaya, Malaysia (UMRG, RP013-2012B grants), the Naval Medical Research Center-Asia (Work Unit Number D1101), and U.S. Department of State, Biosecurity Engagement Program (NAMRU: J-55025-75053). We thank Associate Professor Dmitri Apanaskevich, University of Georgia Southern, for his kind assistance in tick identification.

\section{Author Disclosure Statement}

The authors declare that they have no competing interests. B.L.P. is a military service member of the U.S. Government.
This work was prepared as part of his official duties. The opinions and assertions contained herein are those of the author(s) and are not to be construed as official or reflecting the views of the Department of the Navy, Department of Defense, or the U.S. Government. Title 17 U.S.C. $\$ 105$ provides that "Copyright protection under this title is not available for any work of the United States Government." Title 17 U.S.C. $§ 101$ defines a U.S. Government work as a work prepared by a military service member or employee of the U.S. Government as part of that person's official duties.

\section{References}

Ahantarig A, Malaisri P, Hirunkanokpun S, Sumrandee C, et al. Detection of Rickettsia and a novel Haemaphysalis shimoga symbiont bacterium in ticks in Thailand. Curr Microbiol 2011; 62:1496-1502.

Almeida AP, Marcili A, Leite RC, Nieri-Bastos FA, et al. Coxiella symbiont in the tick Ornithodoros rostratus (Acari: Argasidae). Ticks Tick Borne Dis 2012; 3:203-206.

Arthan W, Sumrandee C, Hirunkanokpun S, Kitthawee S, et al. Detection of Coxiella-like endosymbiont in Haemaphysalis tick in Thailand. Ticks Tick Borne Dis 2015; 6:63-68.

Audy JR, Nadchatram M, Lim BL. Malaysian parasites XLIX. Host distribution of Malayan ticks (Ixodoidea). Stud Inst Med Res Malaya 1960; 225-246.

Bernasconi MV, Casati S, Péter O, Piffaretti J-C. Rhipicephalus ticks infected with Rickettsia and Coxiella in Southern Switzerland (Canton Ticino). Infect Genet Evol 2002; 2: 111-120.

Bina Rai S, Kamaludin F, Chow T, Yoon C. First Documented zoonotic case of $\mathrm{Q}$ fever in Penang, Malaysia. Outbreak Surveil Invest Rep 2011; 4:1-5.

Black WC, Piesman J. Phylogeny of hard- and soft-tick taxa (Acari: Ixodida) based on mitochondrial 16S rDNA sequences. Proc Natl Acad Sci U S A 1994; 91:10034-10038.

Blacksell SD, Kantipong P, Watthanaworawit W, Turner C, et al. Underrecognized arthropod-borne and zoonotic pathogens in Northern and Northwestern Thailand: Serological evidence and opportunities for awareness. Vector Borne Zoonotic Dis 2015; 15:285-290.

Clay K, Klyachko O, Grindle N, Civitello D, et al. Microbial communities and interactions in the lone star tick, Amblyomma americanum. Mol Ecol 2008; 17:4371-4381.

Cooper A, Stephens J, Ketheesan N, Govan B. Detection of Coxiella burnetii DNA in wildlife and ticks in Northern Queensland, Australia. Vector Borne Zoonotic Dis 2012; 13: 12-16.

Drummond AJ, Rambaut A. BEAST: Bayesian evolutionary analysis by sampling trees. BMC Evol Biol 2007; 7:214.

Duron O, Jourdain E, McCoy KD. Diversity and global distribution of the Coxiella intracellular bacterium in seabird ticks. Ticks Tick Borne Dis 2014; 5:557-563.

Duron O, Noël V, McCoy KD, Bonazzi M, et al. The recent evolution of a maternally-inherited endosymbiont of ticks led to the emergence of the $\mathrm{Q}$ fever pathogen, Coxiella burnetii. PLoS Pathog 2015; 11:e1004892.

Duron O, Sidi-Boumedine K, Rousset E, Moutailler S, et al. The importance of ticks in $\mathrm{Q}$ fever transmission: What has (and has not) been demonstrated? Trends Parasitol 2015; 31: 536-552.

Jasinskas A, Zhong J, Barbour AG. Highly prevalent Coxiella sp. bacterium in the tick vector Amblyomma americanum. Appl Environ Microbiol 2007; 73:334-336. 
Khoo JJ, Chen F, Kho KL, Ahmad Shanizza AI, et al. Bacterial community in Haemaphysalis ticks of domesticated animals from the Orang Asli communities in Malaysia. Ticks Tick Borne Dis 2016; 7:929-937.

Klyachko O, Stein BD, Grindle N, Clay K, et al. Localization and visualization of a Coxiella-type symbiont within the Lone Star tick, Amblyomma americanum. Appl Environ Microbiol 2007; 73:6584-6594.

Kurtti TJ, Palmer AT, Oliver JH. Rickettsiella-like bacteria in Ixodes woodi (Acari: Ixodidae). J Med Entomol 2002; 39: 534-540.

Lee J-H, Park H-S, Jang W-J, Koh S-E, et al. Identification of the Coxiella sp. detected from Haemaphysalis longicornis ticks in Korea. Microbiol Immunol 2004; 48:125-130.

Liu L, Li L, Liu J, Hu Y, et al. Coinfection of Dermacentor silvarum Olenev (Acari: Ixodidae) by Coxiella-like, Arsenophonus-like, and Rickettsia-like symbionts. Appl Environ Microbiol 2013; 79: 2450-2454.

Machado-Ferreira E, Dietrich G, Hojgaard A, Levin M, et al. Coxiella symbionts in the Cayenne tick Amblyomma cajennense. Microb Ecol 2011; 62:134-142.

Mediannikov O, Fenollar F, Socolovschi C, Diatta G, et al. Coxiella burnetii in humans and ticks in rural Senegal. PLoS Negl Trop Dis 2010; 4:e654.

Meerburg BG, Reusken CBEM. The role of wild rodents in spread and transmission of Coxiella burnetii needs further elucidation. Wildl Res 2011; 38:617-625.

Meng XJ, Lindsay DS, Sriranganathan N. Wild boars as sources for infectious diseases in livestock and humans. Philos Trans R Soc Lond B Biol Sci 2009; 364:2697-2707.

Moutailler S, Valiente Moro C, Vaumourin E, Michelet L, et al. Co-infection of ticks: The rule rather than the exception. PLoS Negl Trop Dis 2016; 10:e0004539.

Norina L, Sabri Y, Goh MY, Zamri-Saad M, et al. Immunohistological localisation of Coxiella burnetii in various organs of naturally Q-fever infected goats. Pertanika J Trop Agri Sci 2011; 34:167-173.

Paramasvaran S, Sani RA, Hassan L, Krishnasamy M, et al. Ectoparasite fauna of rodents and shrews from four habitats in Kuala Lumpur and the states of Selangor and Negeri Sembilan, Malaysia and its public health significance. Trop Biomed 2009; 26:303-311.

Posada D. jModelTest: Phylogenetic model averaging. Mol Biol Evol 2008; 25:1253-1256.

Reeves W. Molecular evidence for a novel Coxiella from Argas monolakensis (Acari: Argasidae) from Mono Lake, California, USA. Exp Appl Acarol 2008; 44:57-60.

Shivaprasad HL, Cadenas MB, Diab SS, Nordhausen R, et al. Coxiella-like infection in psittacines and a toucan. Avian Dis 2008; 52:426-432.

Silaghi C, Pfister K, Overzier E. Molecular investigation for cacterial and protozoan tick-borne pathogens in wild boars (Sus scrofa) from Southern Germany. Vector Borne Zoonotic Dis 2014; 14:371-373.
Smith TA, Driscoll T, Gillespie JJ, Raghavan R. A Coxiella-like endosymbiont is a potential vitamin source for the Lone Star tick. Genome Biol Evol 2015; 7:831-838.

Špitalská E, Kocianová E. Detection of Coxiella burnetii in ticks collected in Slovakia and Hungary. Eur J Epidemiol 2003; 18:263-266.

Suputtamongkol Y, Rolain J-M, Losuwanaruk K, Niwatayakul $\mathrm{K}$, et al. Q fever in Thailand. Emerging Infect Dis 2003; 9: 1186-1188.

Talavera G, Castresana J. Improvement of phylogenies after removing divergent and ambiguously aligned blocks from protein sequence alignments. Syst Biol 2007; 56:564-577.

Tamura K, Stecher G, Peterson D, Filipski A, et al. MEGA6: Molecular evolutionary genetics analysis version 6.0. Mol Biol Evol 2013; 30:2725-2729.

Tanskul P, Inlao I. Keys to the adult ticks of Haemaphysalis Koch, 1844, in Thailand with notes on changes in taxonomy (Acari: Ixodoidea: Ixodidae). J Med Entomol 1989; 26:573-600.

Tay ST, Ho TM, Rohani MY. Serological findings of Coxiella burnetii infection among patients with fevers in a health centre in Sarawak, Malaysia. Southeast Asian J Trop Med Public Health 1998; 29:94-95.

Tissot-Dupont H, Raoult D. Q Fever. Infect Dis Clin North Am 2008; 22:505-514.

Vapniarsky N, Barr BC, Murphy B. Systemic Coxiella-like infection with myocarditis and hepatitis in an Eclectus parrot (Eclectus roratus). Vet Pathol 2012; 49:717-722.

Wassef HY, Hoogstraal H. Dermacentor (Indocentor) Compactus (Acari: Ixodoidea: Ixodidae): Identity of male and female. J Med Entomol 1983; 20:648-652.

Wassef HY, Hoogstraal H. Dermacentor (Indocentor) Steini (Acari: Ixodoidea: Ixodidae): Identity of male and female. J Med Entomol 1986; 23:532-537.

Watanabe M, Nakao R, Amin-Babjee SM, Maizatul AM, et al. Molecular screening for Rickettsia, Anaplasmataceae and Coxiella burnetii in Rhipicephalus sanguineus ticks from Malaysia. Trop Biomed 2015; 32:390-398.

Zhong J, Jasinskas A, Barbour AG. Antibiotic treatment of the tick vector Amblyomma americanum reduced reproductive fitness. PLoS One 2007; 2:e405.

Address correspondence to: Sazaly AbuBakar Tropical Infectious Diseases Research and Education Centre (TIDREC) Department of Medical Microbiology Faculty of Medicine University of Malaya Kuala Lumpur 50603 Malaysia

E-mail: sazaly@um.edu.my 